\title{
Comparative studies on in vitro sperm decondensation and pro- nucleus formation in egg extracts between gynogenetic and bisexual fish
}

\author{
Chang JiAn LI, JiAn FAng GUI*
}

State Key Laboratory of Freshwater Ecology and Biotechnology, Wuhan Center for Developmental Biology, Institute of Hydrobiology, Chinese Academy of Sciences, Wuhan 430072, China

\begin{abstract}
A cell-free system based upon the egg extracts from gynogenetic gibel carp (Carassius auratus gibelio) or bisexual red common carp (Cyprinus carpio red variety) was developed to investigate developmental behaviors of the demembranated sperm nuclei. Both red common carp and gibel carp sperm nuclei could decondense fully and form pronuclei in the red common carp egg extracts. Gibel carp sperm nuclei could also decondense fully and form pronuclei in the gibel carp egg extracts, but red common carp sperm nuclei could not decondense sufficiently in the same extracts. The significant differences of morphological changes were further confirmed by ultrastructural observation of transmission electron microscopy. The data further offer cytological evidence for gonochoristic reproduction in the gynogenetically reproducing gibel carp. In addition, the sperm nuclei in vitro decondensation is dependent on the $\mathrm{pH}$ in the extracts, and the decondensed efficiency is optimal at $\mathrm{pH}$ 7. However, no DNA replication was observed in the two kinds of egg extracts during the incubation period of the sperm nuclei. It is suggested that the egg extracts prepared from the gynogenetic gibel carp should be a valid in vitro system for studying molecular mechanism on gynogenesis and reproduction mode diversity in fish.
\end{abstract}

Key words: decondensation, pronucleus formation, cell-free system, gynogenesis, gibel carp.

\section{INTRODUCTION}

During maturation of most animal sperm cells, sperm-specific nuclear proteins replace somatic histones, and the chromatin becomes highly compacted in preparation for the sperm journey to the egg[1]. This nuclear condensation reverses rapidly following sperm-egg membrane fusion and sperm nuclear incorporation into the egg during fertilization. The sperm nuclear decondensation is obviously a critical early step in embryonic developmental process, necessary for duplication and ultimate transcription of the male genome[2]. Plenty of

\footnotetext{
* Correspondence: Prof. Jian Fang GUI

Fax: 0086-27-87875132 (fax); E-mail: jfgui@ihb.ac.cn

Received Dec-18-2002 Revised Mar-10-2003 Accepted Apr-112003
}

information on the early events of sperm nuclei after fertilization, including swelling, chromatin decondensation, DNA synthesis, and nuclear envelope assembly, have been obtained from the studies in cell-free systems based on Xenopus eggs extracts [3-6], and cytosol extracts of Drosophila embryo[7], CHO cells[8], sea urchin eggs[9], and carrot cell[10].

Fishes, the biggest and most diverse community in vertebrates, are good experimental models for studies on cell and developmental biology by many favorable characteristics[11]. However, studies on the molecular mechanisms of fish sperm decondensation process has been difficult because it is hard to develop a nice cell-free system based on fish eggs for the performance of in vitro biochemical 
studies. Most of the studies on early developmental events of sperm nuclei after fertilization in fish were based on the methods of microinjection together with cytological and ultrastructural observation[12-14]. It is necessary to develop a nice cell-free system to study the sperm decondensation and pronucleus formation in fish.

It has been demonstrated in bisexual reproduction fish that sperm nucleus undergoes nuclear envelope breakdown, nucleus swelling, and pronuclear formation with a new nuclear envelope after fertilization $[12,15]$. In contrast to common bisexual reproduction fish, within the fertilized eggs of gibel carp (Carassius auratus gibelio), the heterologous sperm nucleus keeps in condensation and is failed to form male pronucleus, while some of the homologous sperm nuclei can decondense and transform into male pronucleus. And there are about 5-20\% males in natural populations of gibel carp[16]. Therefore, the gynogenetic gibel carp has been considered as a unique system for studying mechanism of oocyte maturation, sex determination, and early embryonic development[17-21]. However, how to explain the difference of early developmental behaviors between the homologous sperm and heterologous sperm in the gibel carp egg? What is the molecular mechanism by which the natural gynogenesis is regulated in gibel carp? In order to answer the two fascinating questions, an in vitro experimental system requires to be established. In this study, a cell-free system based upon the egg extracts from the gynogenetic gibel carp or bisexual red common carp is developed to investigate developmental behaviors of the demembranated sperm nuclei.

\section{MATERIALS AND METHODS}

\section{Obtainment of eggs and preparation of egg extracts}

The adult fishes, gynogenetic gibel carp (Carassius auratus gibelio) and bisexual red carp (Cyprinus carpio) red variety) were obtained from Guanqiao Experimental Station, Institute of Hydrobiology, Chinese Academy of Sciences. Eggs were obtained by two intraperitoneal injections with a mixture of HCG and LRHA into the adult females. HCG and LRH-A were purchased from Shanghai Lizhu Dong Feng Biotechnology Co., LTD. The first injection was $600 \mathrm{IU}$ HCG and $6 \mu \mathrm{g} \mathrm{LRH}-\mathrm{A} / \mathrm{kg}$ fish weight. The second injection was $200 \mathrm{IU}$ HCG and $2 \mu \mathrm{g}$ LRH-A/kg fish weight. The interval between two injections was $8 \mathrm{~h}$. About $8 \mathrm{~h}$ after the second injection, eggs were obtained from the females. The eggs extracts were prepared essentially as described by Blow and Laskey with some modifications[22]. Briefly, eggs were rinsed 3 times using $1 / 4$ times MMR buffer $(100 \mathrm{~m} M \mathrm{NaCl}, 2 \mathrm{mM} \mathrm{KCl}, 1 \mathrm{mM} \mathrm{MgCl}$, $2 \mathrm{mM} \mathrm{CaCl}, 0.1 \mathrm{mM}$ EGTA, and $5 \mathrm{~m} M$ HEPES, $\mathrm{pH}$ 7.8), then dejellied in MMR buffer containing $2 \%$ cysteine ( $\mathrm{pH} 7.8$ ). The eggs were then rinsed 3 times, and the decondensing activity was induced by adding calcium ionophore A23187 (10 $\mu \mathrm{M}$ in DMSO; Sigma Chemical Co.) to a final concentration of $100 \mathrm{n} M$ for $15-20$ min. After rinsing with $\mathrm{MMR}$ containing $2 \mathrm{mM} \mathrm{MgCl}$, and $0.6 \mathrm{mM}$ $\mathrm{CaCl}_{2}$, the eggs were washed with lysate buffer ( $250 \mathrm{mM}$ sucrose, $50 \mathrm{~m} M \mathrm{KCl}, 2.5 \mathrm{mM} \mathrm{MgCl}, 10 \mathrm{~m} M$ HEPES, $\mathrm{pH}$ 7.4) and the lysate buffer was removed as much as possible by centrifugation at 500 g. The eggs were homogenized gently in a homogenizer in the presence of several protease inhibitors (aprotinin and leupeptin, final concentration $10 \mu \mathrm{g} / \mathrm{ml}$ ). The homogenates were subjected to centrifugation for $15 \mathrm{~min}$ at 9,000 g and were separated into three layers: a white lipid upper layer, a clear yellow middle layer and a viscous pellet. After removing the lipid layer, the yellow middle layer was collected with a chilled pipette and was clarified by recentrifugation. The resulting cytoplasmic supernatant was recovered and either used freshly or frozen for future use.

\section{Preparation of demembranated sperm nuclei}

Demembranated sperm nuclei were prepared using a modification of the method described by Philpott et al[4]. Male fishes were injected with 500 IU HCG $/ \mathrm{kg}$ fish weight. About $12 \mathrm{~h}$ later, the sperm were obtained from the injected male fishes and washed 4 times in buffer XN [50 mM HEPES-KOH (pH 7.0), 250 $\mathrm{mM}$ sucrose, $75 \mathrm{~m} M \mathrm{NaCl}, 0.5 \mathrm{~m} \mathrm{M}$ spermidine, $0.15 \mathrm{~m} M$ spermine]. Washed sperm were resuspended in $500 \mu \mathrm{l}$ of XN buffer. To demembranate sperm, $100 \mu \mathrm{l}$ of $2 \mathrm{mg} / \mathrm{ml}$ lysolecithin (Sigma) in buffer XN was added followed by incubation on ice for $10 \mathrm{~min}$. Permeabilization was stopped by adding $1 \mathrm{ml}$ of $3 \%$ bovine serum albumin (BSA) in buffer XN. Sperm nuclei were washed three times in buffer XN/0.4\% BSA, and then finally resuspended in buffer XN/30\% glycerol. Sperm nuclei were counted with a hemocytometer, and stored at $-7^{\circ} \mathrm{C}$.

\section{In vitro decondensation and fluorescent microscopy}

The eggs extracts were adjusted to $\mathrm{pH} 7.0$ or other $\mathrm{pH}$ value with $50 \mathrm{~m} M$ Hepes ( $\mathrm{pH} 7.8$ ) and $0.1 \mathrm{~N} \mathrm{NaOH}$ or $\mathrm{HCl}$. The demembranated sperm was incubated at $23^{\circ} \mathrm{C}$ in $200 \mathrm{ml}$ egg extracts containing an ATP regenerating system ( $2 \mathrm{~m} M$ ATP, $20 \mathrm{~m} M$ phosphocreatine, and $50 \mu \mathrm{g} / \mathrm{ml}$ creatine kinase). Samples were mixed gently. $5 \mu \mathrm{l}$ aliquots were sampled at each time point, and mixed with $10 \mu \mathrm{l}$ solution containing $1 \mu \mathrm{g} / \mathrm{ml}$ Hoechst 33258 and $1 \%$ formaldehyde. Aliquots were then viewed wet under a Leica DMIRB inverted microscope. Images were captured with a Leica DC100 image analyzer. Three fields from the same slide, containing 5-40 nuclei/ field, were counted for determing the percentage of decondensation present at a given time.

\section{Transmission electron microscopy}

Samples for electron microscopy were fixed by overlaying with $800 \mu$ l of cold $2.5 \%$ glutaraldehyde in PBS (137 mM NaCl, $2.7 \mathrm{mM}$ $\mathrm{KCl}, 10 \mathrm{mM} \mathrm{Na}{ }_{2} \mathrm{HPO} 4,2 \mathrm{mM} \mathrm{KHPO}_{4}, \mathrm{pH} 7.4$ ), and kept at $4^{\circ} \mathrm{C}$ overnight. The samples were spun at $2,500 \mathrm{~g}$ for $5 \mathrm{~min}$ and the pellets washed three times with PBS. Pellets were postfixed for $3 \mathrm{~h}$ at $4^{\circ} \mathrm{C}$ using $1 \% \mathrm{OsO}_{4}$ in $\mathrm{PBS}$, then further washed three times in 
this buffer before block staining overnight in $2 \%$ uranyl acetate. The samples were dehydrated through ethanol and embedded in polymerized acrylic resin. Blocks were sectioned to give silver to pale gold sections that were taken onto collodion- and carbon- coated 600-mesh copper grids before double staining using uranyl acetate and Reynold's lead citrate (5 min each). The sections were viewed using a transmission electron microscope (JEM-1230) at $80 \mathrm{kV}$.

\section{Flow cytometry analysis}

For flow cytometric analysis, the demembranated sperm nuclei were prepared at different interval according to the method described by Blow and Watson[23]. The isolated nuclei were stained with $1 \mathrm{ml}$ of a solution of $5 \mu \mathrm{g} / \mathrm{ml}$ propidium iodide and $50 \mu \mathrm{g} / \mathrm{ml}$ RNase. The above nuclei were analysed for DNA with the Coulter Epics Altra Flow Cytometer (Beckman Coulter Co., USA.). The laser was tuned to $488 \mathrm{~nm}$ line at a light power, which excites red fluorescence from the propidium iodide/DNA complex.

\section{RESULTS}

Similarity of morphological changes between red common carp sperm nuclei and gibel carp sperm nuclei in the egg extracts from red common carp

Incubated in the egg extracts from red common carp, both red common carp and gibel carp demembranated sperm nuclei underwent chromatin decondensation and similar morphological changes (Fig 1). The two kinds of sperm nuclei were highly condensed when added into the egg extracts from red common carp, and the gibel carp sperm nuclei are larger than the red common carp sperm nuclei (Fig 1A-a, B-a). At 30 min of incubation, 49. $4 \%$ of the red carp sperm nuclei transformed into decondensed forms including forms of swollen conical, elongated bar, and swollen sphere (Fig 1Ac, Fig 2A). At $60 \mathrm{~min}$ after incubation, the decondesed nuclei increased to over $80 \%$. At that time, these sperm nuclei had decondensed to form small pronuclei (Fig 1A-d), and the pronuclei further enlarged at $90 \mathrm{~min}$ (Fig $1 \mathrm{~A}-\mathrm{e}$ ). Up to $120 \mathrm{~min}$ after incubation, the decondesed nuclei reached to $90.5 \%$ (Fig 1A-f, Fig 2A). Similarly, $44.0 \%$ of the demembranated sperm nuclei of gibel carp were decondensed at $30 \mathrm{~min}$ of incubation in the egg extract of red common carp. And they began to transform their shape from condensed small sphere to long bar or conical shape after $60 \mathrm{~min}$. Moreover, the pronuclei were formed as similar to that of red carp sperm. The percentage of the decondensed sperm nuclei was $88.5 \%$ at $120 \mathrm{~min}$ of incubation (Fig 1B, Fig 2A).
Difference of morphological changes between red common carp sperm nuclei and gibel carp sperm nuclei in the egg extracts from gynogenetic gibel carp

When incubated in the egg extracts of gynogenetic gibel carp, the sperm nuclei of red carp were kept in condensed state or only underwent subtle swollen change during the incubation (Fig 3A), whereas the gibel carp sperm nuclei exhibited obviously morphological changes (Fig 3B). The typically decondensed changes and pronucleus formation (Fig 3B) were similar to that of red common carp sperm and gibel carp sperm in the egg extracts from red common carp. When introduced into the egg extract from gibel carp and observed immediately under fluorescent microscope, $83.8 \%$ of the demembranated gibel carp sperm nuclei displayed a condensed sphere. By 5 min of incubation, $46 \%$ of the sperm nuclei began to elongate, swell, and decondense. After incubation for $60 \mathrm{~min}$, about $75 \%$ of the nuclei were decondensed, and the decondensed nuclei reached to $85.1 \%$ after incubation for $120 \mathrm{~min}$ (Fig $2 \mathrm{~B}$ and Fig 3B). However, when incubated in the same egg extracts, most of red carp sperm nuclei did not show obviously morphological changes. No decondensed nucleus was observed during the whole incubation period (Fig 3A).

\section{Pronucleus formation of gibel carp sperm nuclei in the egg extracts from gynogenetic gibel carp}

Pronucleus formation was observed in both red common carp and gibel carp sperm nuclei after incubated in the egg extracts of red common carp for more than $60 \mathrm{~min}$ (Fig 1), and similar process of pronucleus formation was also revealed in gibel carp sperm nuclei after incubated in the egg extracts of gibel carp for about same time (Fig 3B). Under high magnification (1000 times), it was observed clearly that the chromatin of these decondensed sperm nuclei was loosed and with less density (Fig 4 a-d). Furthermore, we also observed that some of them transformed into decondensed swollen spheres whose chromation was obviously loosed and the fluorescent intensity was also weakened, showing that in vitro male pronucleus formation was occurred in the above cell-free system (Fig $4 \mathrm{~d}$ ), and that the pronucleus formation in vitro was similar to that in vivo observed previously in bisexual fish eggs[14]. However, no male pronucleus formation was ob- 
In vitro sperm decondensation and pronucleus formation in fish
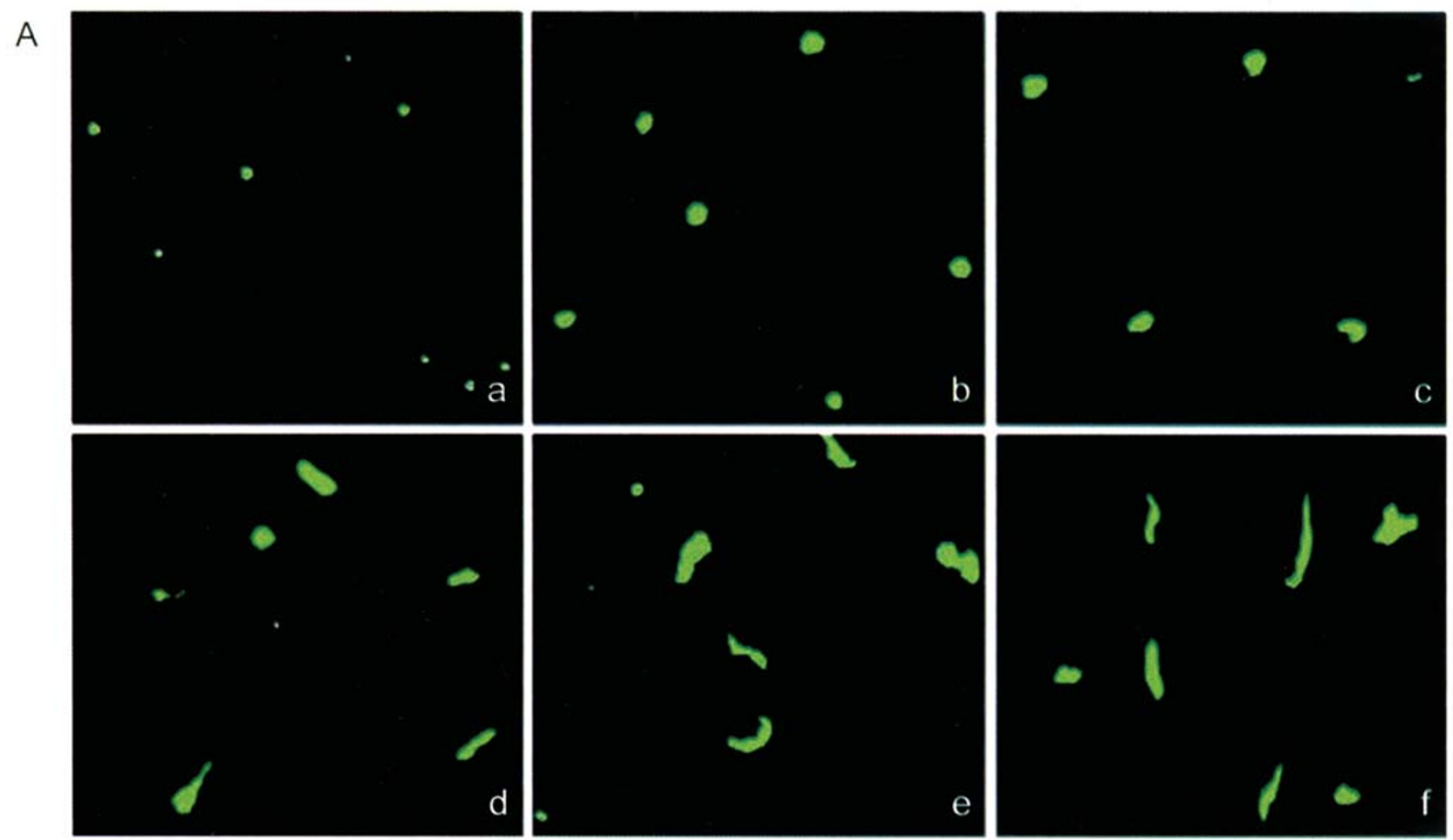

B
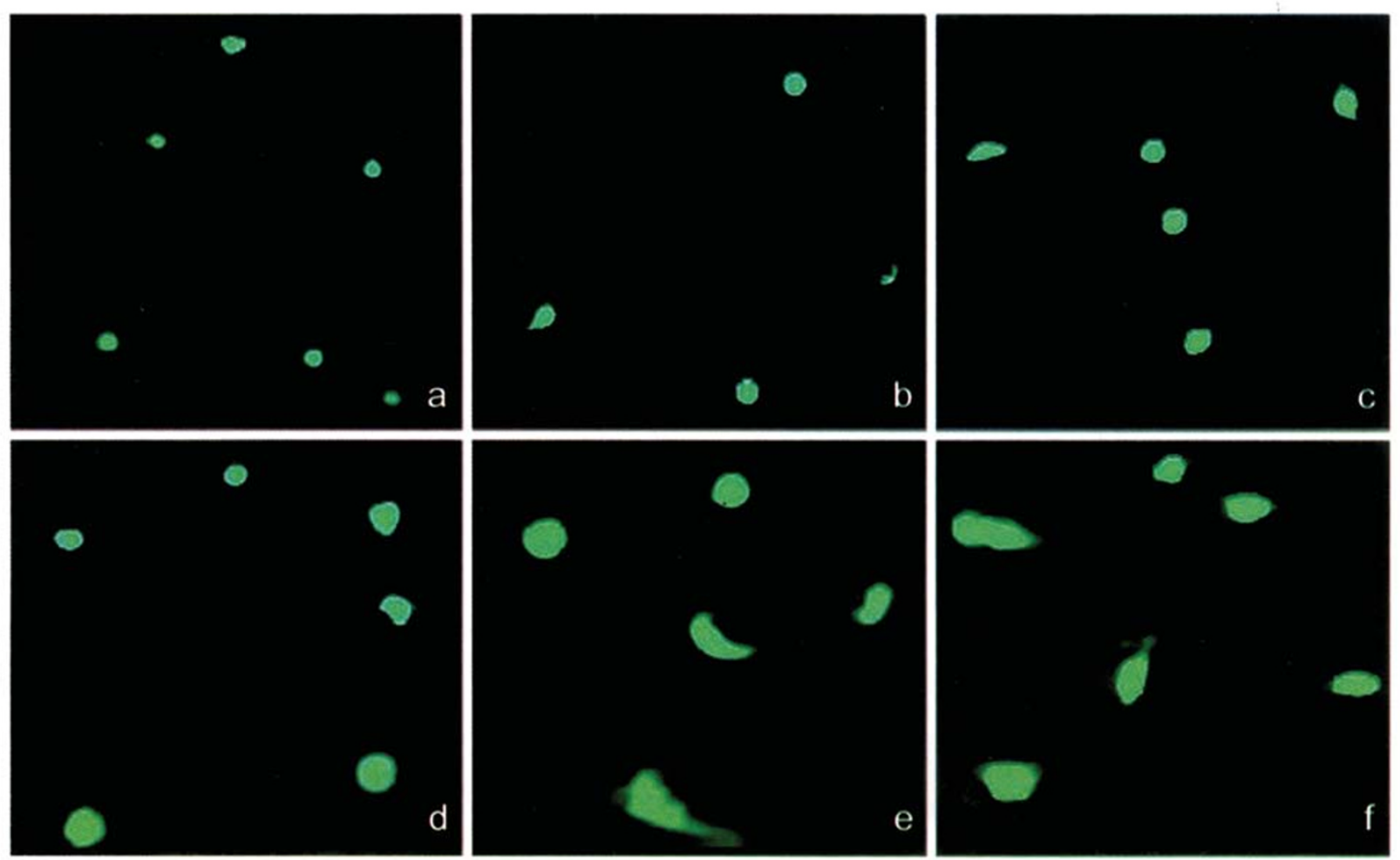

Fig 1. Decondensation process of demembranated sperm nuclei in red common carp egg extracts $(200 \times)$. (A) Red common carp sperm nuclei; (B) Gibel carp sperm nuclei. The sperm nucleus samples incubated at (a) 0 min, (b) 5 min, (c) $30 \mathrm{~min}$, (d) $60 \mathrm{~min}$, (e) $90 \mathrm{~min}$, and (f) $120 \mathrm{~min}$ were collected and observed by fluorescent microscopy. 

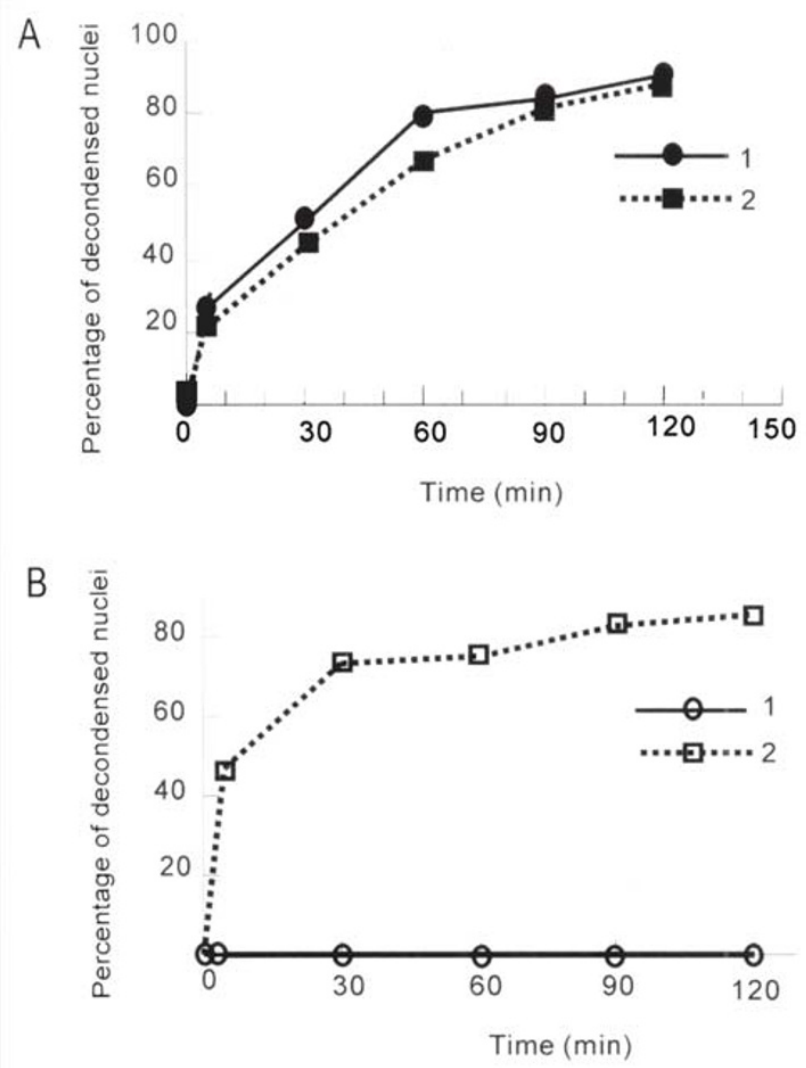

Fig 2. Percentage of decondensed sperm nuclei in red common carp egg extracts $(\mathbf{A})$ and gibel carp egg extracts $(\mathbf{B})$. 1. Red common carp sperm nuclei; 2. Gibel carp sperm nuclei.

served when red carp demembranated sperm nuclei were introduced into the eggs extract of gibel carp for $120 \mathrm{~min}$ (Fig 3A).

Ultrastructural changes of red common carp sperm nuclei and gibel carp sperm nulei in the egg extracts from gynogenetic gibel carp

In order to further investigate the structural and morphological differences of gibel carp or red common carp sperm nuclei following incubation in the egg extracts from gynogenetic gibel carp, transmission electron microscopy were applied to ultrastructural investigation. As shown in Fig 5, the gibel carp sperm nuclei obviously enlarged, and the chromatin dispersed after one-hour incubation in the egg extracts from gibel carp (Fig 5A-b, 5B-b). However, no obvious change in size, morphology or chromatin decondensation was observed when the demembranated red carp sperm nuclei were introduced into the same cell-free system and incubated for the same time (Fig 5A-d, 5B-d).

DNA content analysis on different decondensation sperm nuclei by flow cytometry

DNA contents of the two kinds of sperm nuclei incubated for different times in the two egg extracts were analyzed with flow cytometer to investigate whether DNA replication was occurred during sperm nuclei decondensation in vitro. As show in Fig 6, no change in the DNA contents was observed in all the samples during incubation of $300 \mathrm{~min}$, suggesting that no DNA replication was performed in the two kinds of egg extracts during the incubation period of the sperm nuclei.

\section{Effect of $\mathrm{pH}$ on sperm decondensation}

In some animal species, fertilization often results in an increase of cytoplasmic $\mathrm{pH}$ from approximately 6.3 to 7.2 in vivo[24, 25]. In order to optimize $\mathrm{pH}$ in the cell-free system, we tested the effects of $\mathrm{pH}$ on the decondensation of demembranated sperm nuclei. As shown in Fig 7, the decondensed efficiency is optimal at $\mathrm{pH} 7$ whether the extracts are from red common carp eggs or from gibel carp eggs.

\section{DISCUSSION}

As shown in the present study, a cell-free system has been established on the basis of the egg extracts from bisexual red common carp and gynogenetic gibel carp, and significant differences of morphological changes are revealed by fluorescent microscopy and transmission electron microscopy between demembranated red common carp sperm nuclei and gibel carp sperm nuclei in the egg extracts from gynogenetic gibel carp. The red carp sperm nuclei are kept in condensed state in egg extracts from the gynogenetic gibel carp (Fig 3A), whereas the gibel carp sperm nuclei in the gibel carp extracts undergo obviously morphological changes, such as full decondensation and pronucleus formation (Fig 3B) that are similar to that of red common carp sperm and gibel carp sperm in the egg extracts from bisexual red common carp (Fig 1). The data further offer cytological evidence for gonochoristic reproduction in the gynogenetically reproducing gibel carp. Consistent with the Previous studies on molecular markers[21, 26] and karyotypic diversity [27], the two kinds of differently developmental behaviors be- 
In vitro sperm decondensation and pronucleus formation in fish

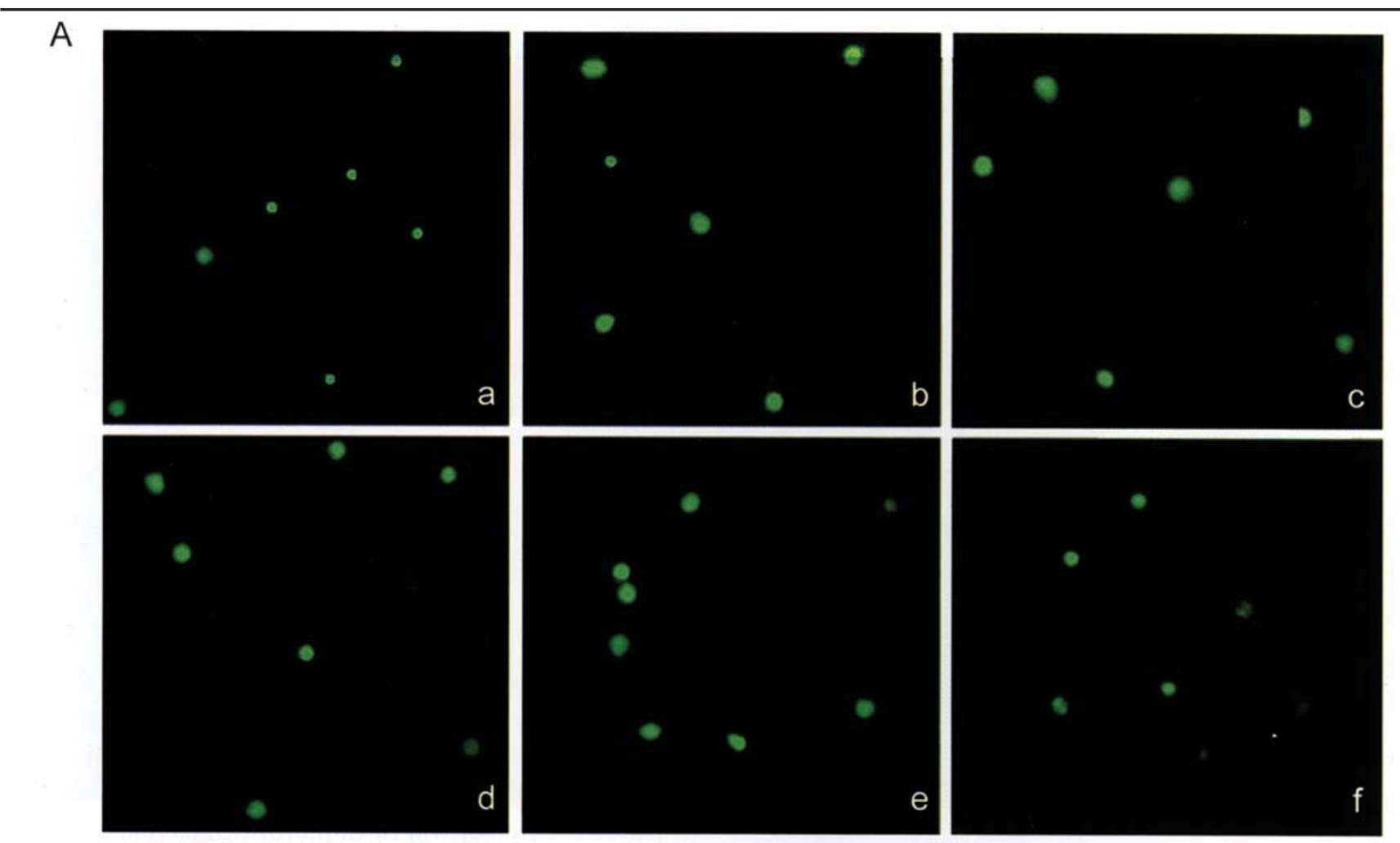

\section{B}
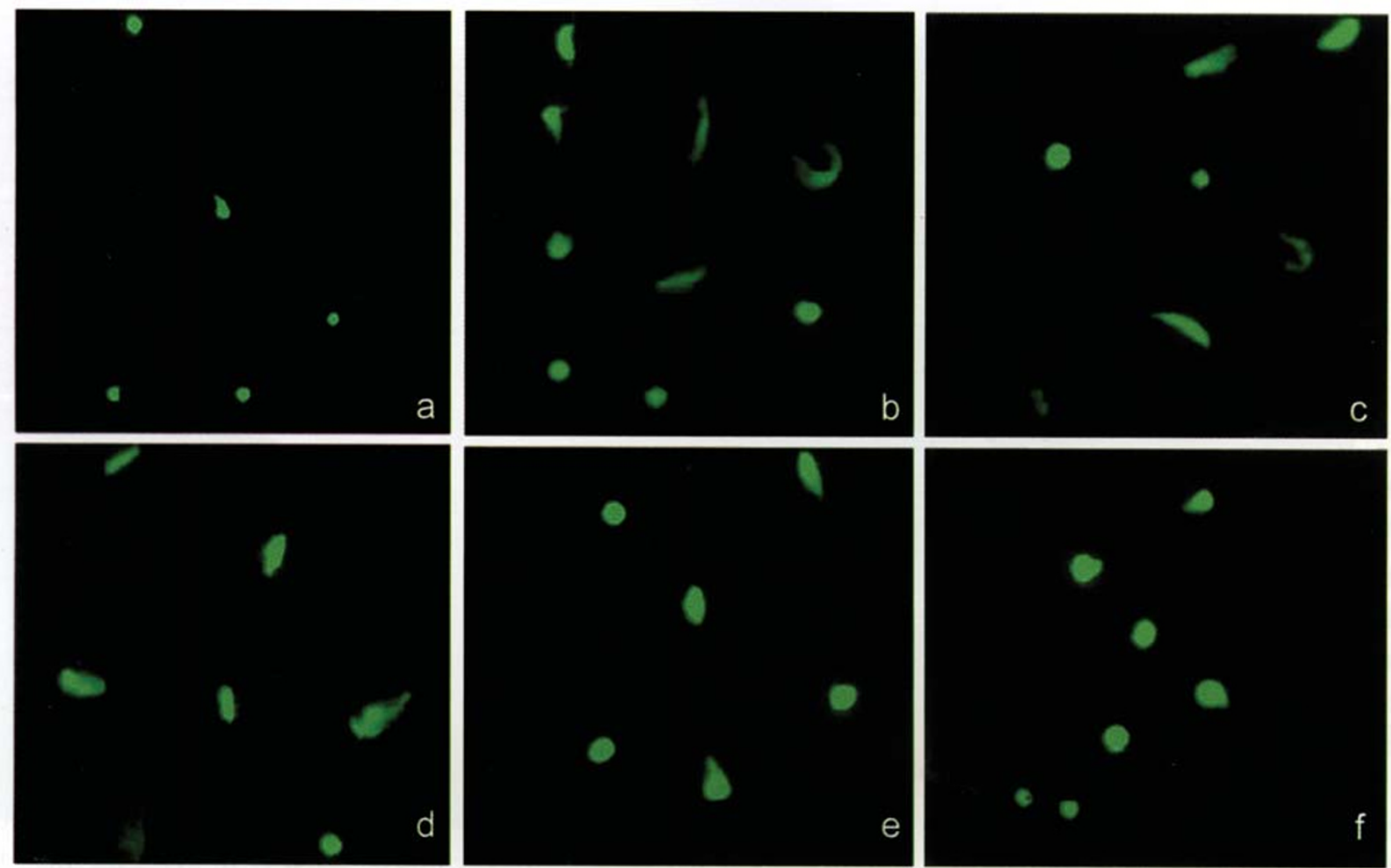

Fig 3. Decondensation process of demembranated sperm nuclei in gibel carp egg extracts $(200 \times)$. (A) Red common carp sperm nuclei; (B) Gibel carp sperm nuclei. The sperm nucleus Samples incubated at (a) 0 min, (b) 5 min, (c) 30 min, (d) 60 min, (e) $90 \mathrm{~min}$, and (f) 120 min were collected and observed by fluorescent microscopy. 

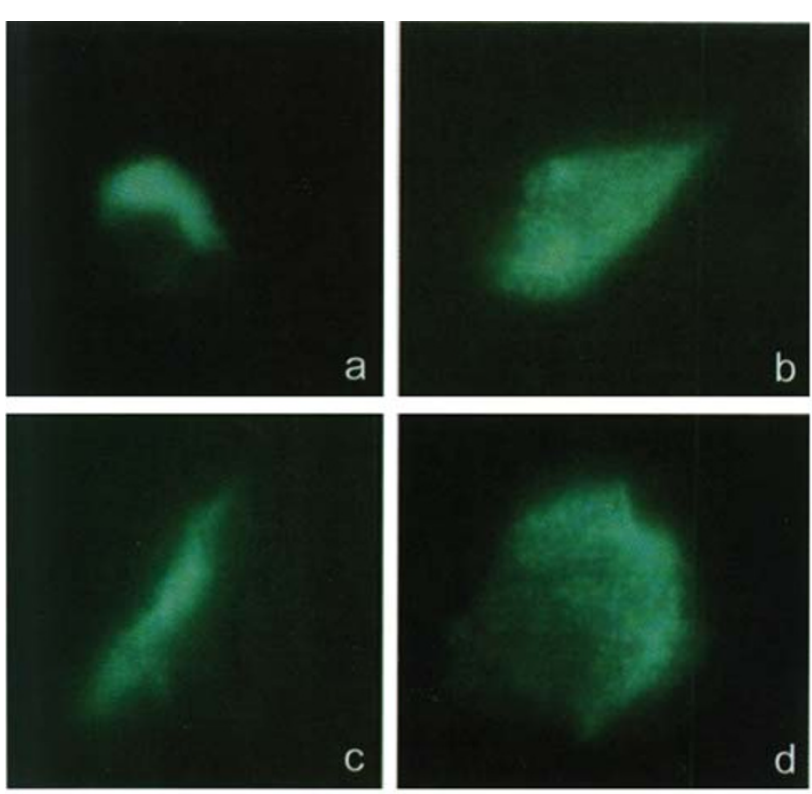

Fig 4. Several typical pronucleus morphology (a-arc, bconical, c-long bar, d-swollen sphere) observed in the incubation group of gibel carp sperm nuclei in the gibel carp egg extracts $(1000 \times)$.

tween red common carp sperm nuclei and gibel carp sperm nuclei in the egg extracts from gynogenetic gibel carp should be the response mechanism of two reproductive development modes, i.e., gynogenetic and gonochoristic reproduction. Thus, the cell-free system prepared from the gibel carp eggs can mimic the developmental processes occurred in fertilization in vivo, and further confirms that the gibel carp posseses two reproductive modes.

Previous investigations in many bisexual species had demonstrated that the sperm decondensation in cell-free system was not species-specific[28-30]. And some heterologous sperm nuclei with far relationship to the species from which the extract were made could be decondensed in the Xenopus laevis egg extract or cell-free extract of carrot[10, 31]. We observed that both the red carp and gibel carp sperm nuclei incubated in the red carp egg extracts decondensed and swelled obviously, and underwent pronucleus formation (Fig 1). However, no red carp sperm nuclei could decondense fully after incubation for $90 \mathrm{~min}$ or even $120 \mathrm{~min}$ in the gibel carp egg extracts, whereas more than $80 \%$ of gibel carp sperm nuclei underwent deconsendation and pronucleus formation at the same time point in the same egg extracts (Fig 2B, Fig 3A and 3B). This phenom- enon implies that different sperm nuclei incubated in the gibel carp egg extracts undergo different developmental process.

After fertilization in vivo in bisexual fish, the membrane of egg was fused with sperm membrane. And a series of physiological changes including the activation of some factors required for the collapse of sperm nuclear membrane were occurred after the fusion, which was a prerequisite for the decondensation of sperm nucleus in vivo. Different from observation in vivo, it was observed that both intact red carp sperm nucleus and gibel carp sperm nucleus could not be decondensed in the above two kinds of egg extracts (data was not shown), which indicated that the collapse of sperm nuclear membrane was required for the decondensation of sperm nucleus in vitro. We speculated that the above difference was resulted from the inactivation of some factors required for the collapse of sperm nuclear membrane.

The formation of male pronucleus is a prerequisite for the fusion of male pronucleus and female pronucleus. In the gibel carp egg extracts, the demembranated gibel carp sperm nuclei were able to form male pronuclei with loose chromatin and weak fluorescent intensity after $120 \mathrm{~min}$ of incubation, whereas no male pronucleus formation was observed when the demembranated red common carp sperm nuclei were introduced into the same system at the same time point. In contrast, both red common carp and gibel carp sperm nuclei were able to transform into male pronuclei in the red common carp egg extracts after $120 \mathrm{~min}$ incubation. The difference of male pronucleus formation between gibel carp and red common carp sperm nuclei in the gibel carp egg extracts is likely related to the mechanism underlying different reproduction modes. Therefore, we propose that some regulative factors might exist in the gibel carp egg cytoplasm. A series of molecular and biochemical studies on identification of the candidate factors have been initiated in our laboratory[32-36], and the studies will help us to elucidate the molecular basis underlying the reproduction modes.

In the cell-free system of Xenopus eggs, 70\%$100 \%$ of Xenopus sperm nuclei had been demonstrated to replicate completely under optimal conditions[22]. DNA replication initiation of sperm nu- 
A
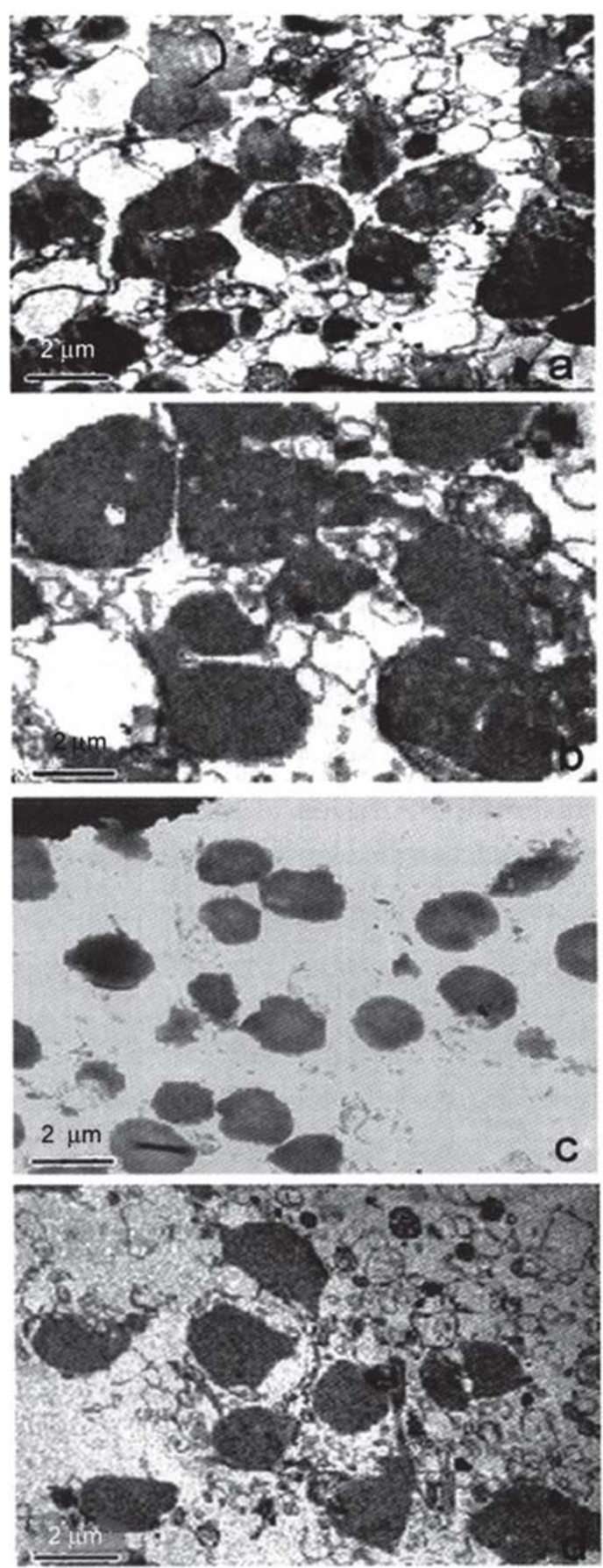

B
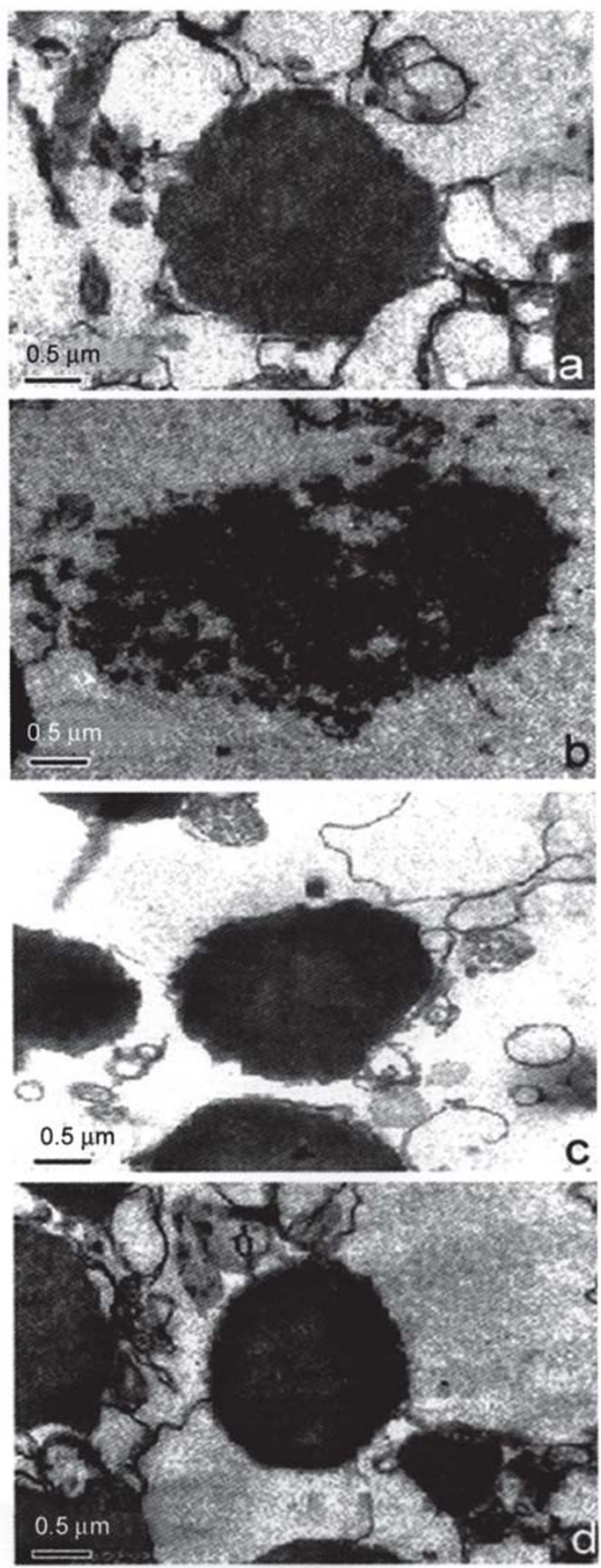

Fig 5. Electron micrographs $(A, 8,000 \times$; B, 20,000 $\times$ ) of condensed and decondensed sperm nuclei. (a) the condensed gibel carp sperm nuclei before incubation, (b) the decondensed gibel carp sperm nuclei incubated in the gibel carp egg extracts for 60 min, (c) the condensed red common carp sperm nuclei before incubation, (d) the condensed red common carp sperm nuclei incubated in the gibel carp egg extracts for $60 \mathrm{~min}$. 

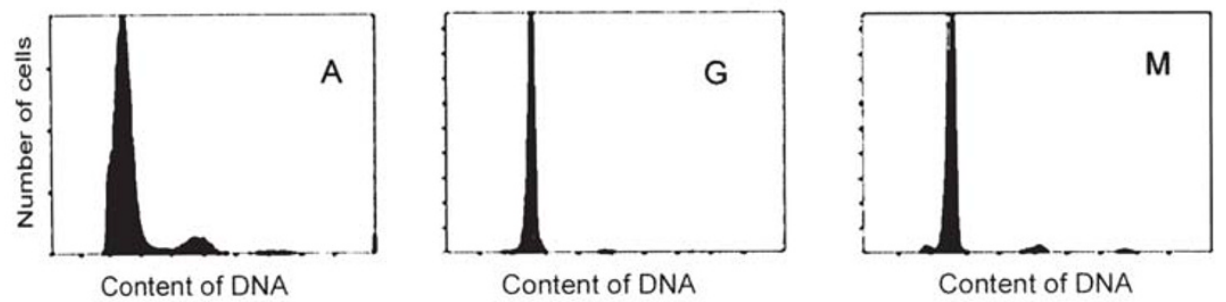

Content of DNA

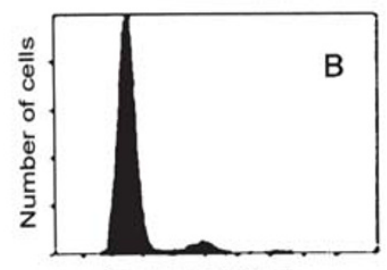

Content of DNA
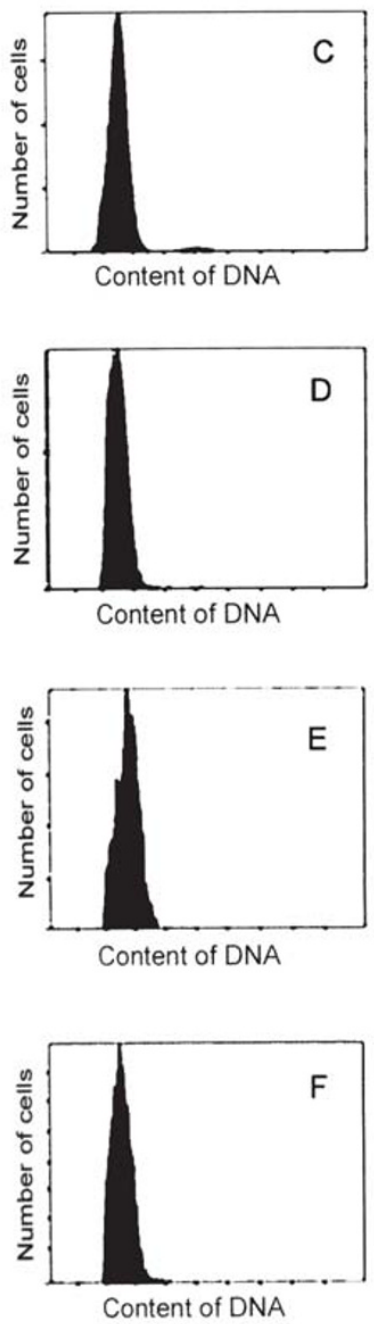

Content of DNA

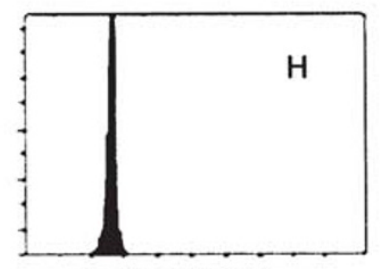

Content of DNA
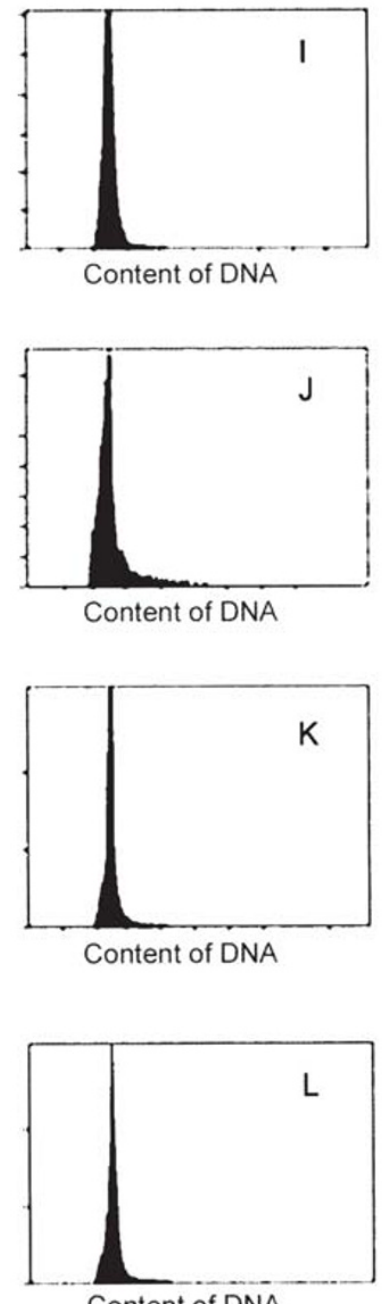

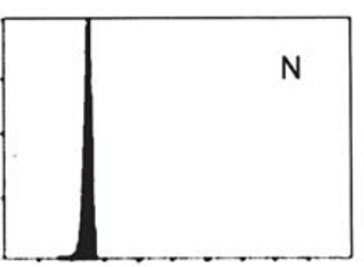

Content of DNA

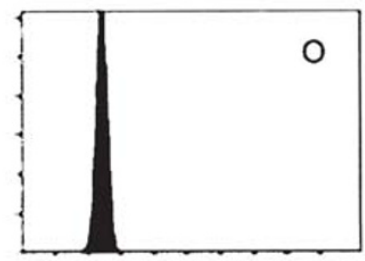

Content of DNA

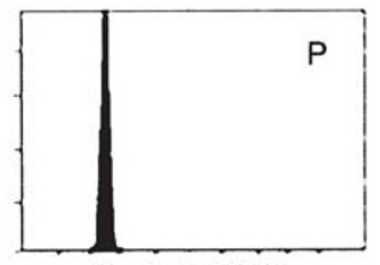

Content of DNA

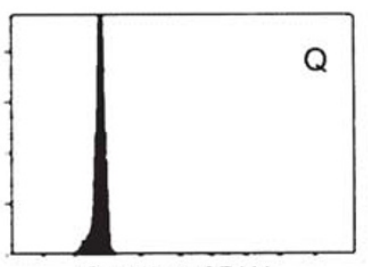

Content of DNA

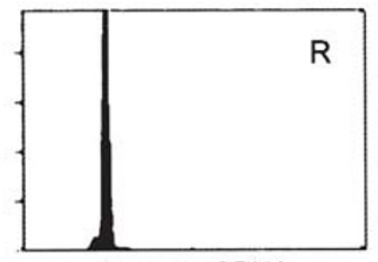

Content of DNA

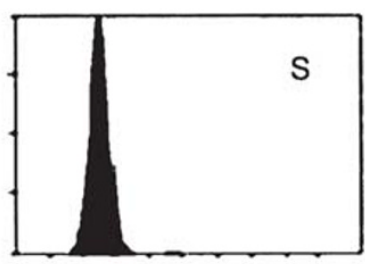

Content of DNA

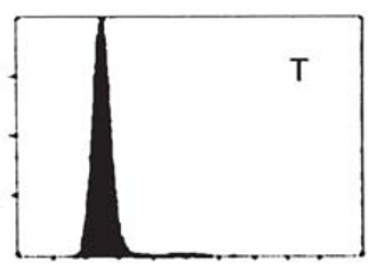

Content of DNA

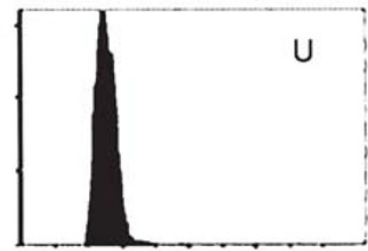

Content of DNA

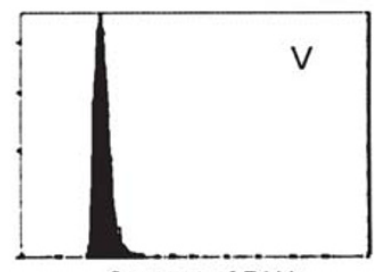

Content of DNA
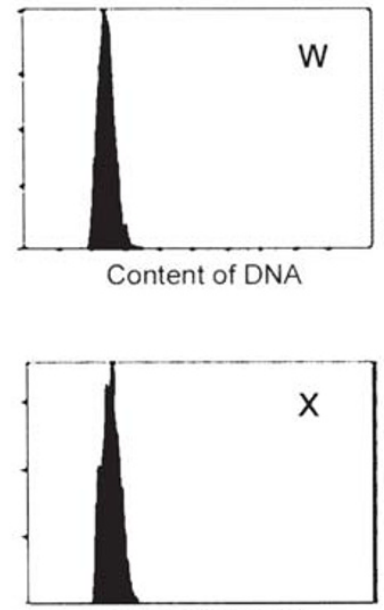

Content of DNA

Fig 6. DNA content detection of sperm nuclei in egg extracts. (A-F) gibel carp sperm nuclei in gibel carp egg extracts, (G-L) red common carp sperm nuclei in gibel carp egg extracts, (M-R) red common carp sperm nuclei in red common carp egg extracts, (S-X) gibel carp sperm nuclei in red common carp egg extracts. Samples were collected at 0 h (A, G, M, S), 1 h (B, H, $\mathrm{N}, \mathrm{T}), 2 \mathrm{~h}(\mathrm{C}, \mathrm{I}, \mathrm{O}, \mathrm{U}), 3 \mathrm{~h}(\mathrm{D}, \mathrm{J}, \mathrm{P}, \mathrm{V}), 4 \mathrm{~h}(\mathrm{E}, \mathrm{K}, \mathrm{Q}, \mathrm{W})$, and $5 \mathrm{~h}(\mathrm{~F}, \mathrm{~L}, \mathrm{R}, \mathrm{X})$, and detected with flow cytometry as described in materials and methods. 

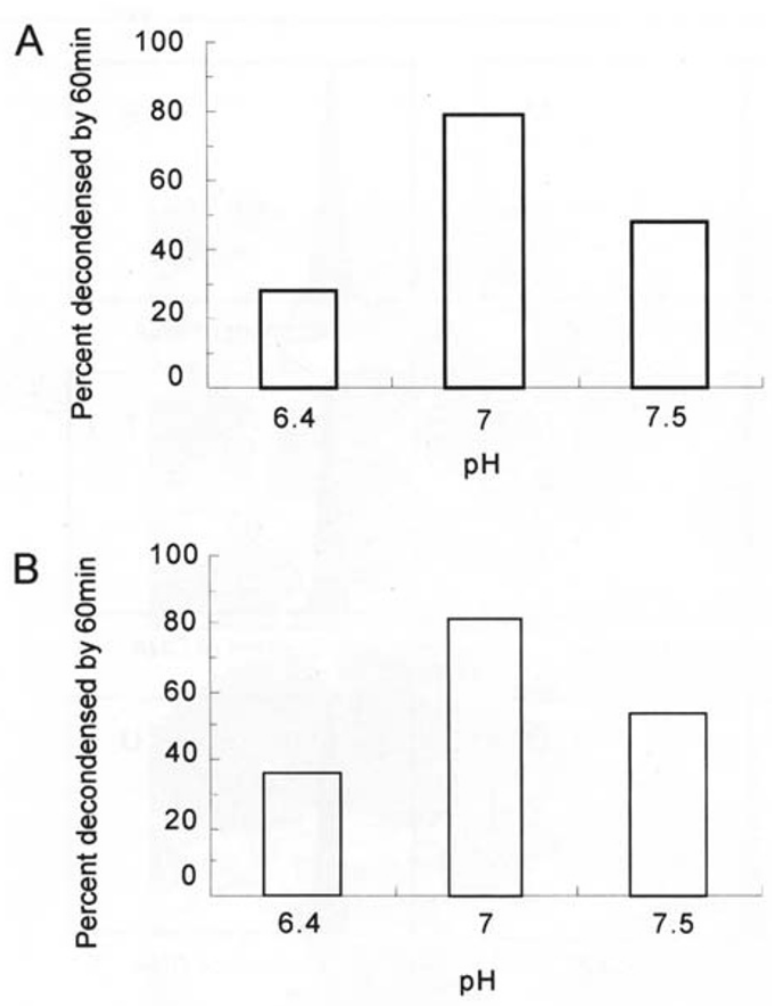

Fig 7. Effect of pH on sperm nuclei decondensation in cell-free system. (A) Gibel carp sperm nuclei incubated in gibel carp egg extracts. (B) Red common carp sperm nuclei incubated in red common carp egg extracts.

clei was also observed in a human cell-free system [37]. However, no DNA replication was observed during the incubation period of the sperm nuclei in the two kinds of egg extracts. Sheehan et al had isolated the Xenopus egg extract into different fractions required for chromatin decondensation and DNA replication respectively[38], indicating that chromatin decondensation and DNA replication were two different processes and that they required different factors. Moreover, some factors related to the initiation or activiation of DNA replication had been identified[39]. The reason that no DNA replication was observed in the fish cell-free system might be that the factors required for initiating the DNA replication lacked or inactivated in the extracts. The formation of male pronucleus was judged by some morphological changes which were occurred after sperm nucleus was introduced into the cell-free extract in previous studies[40]. Therefore, we considered that the male pronucleus was formed after some morphological transformations of the sperm nucleus were happened in the fish cell-free system in spite of the absence of DNA replication.

In some species, fertilization often results in an increase of cytoplasic $\mathrm{pH}$ from approximately 6.3 to 7.2 in vivo[24, 25]. And the optimal $\mathrm{pH}$ for sperm nucleus decondensation in cell-free system from sea urchin eggs is 7.0[9]. As shown in Fig 7, response of our system was also optimal at $\mathrm{pH}$ 7.0, confirming that $\mathrm{pH}$ could play important effect on sperm nucleus decondensation in cell-free system.

Although no DNA replication is observed in the cell-free system reported here, it can mimic the process of sperm nuclei decondensation after fertilization in vivo. It will allow experiments with immunodepletion or biochemical studies otherwise difficult or impossible to perform in vivo. Such experiments should facilitate to elucidate molecular mechanisms underlying reproductive mode diversity of gibel carp. Therefore, the egg extract should be a valid in vitro system for studying the mechanism.

\section{ACKNOWLEDGMENTS}

This work was supported by grants from the National Natural Science Foundation of China (No. 30130240 and 30070379), the Project of Chinese Academy of Sciences (KSCX2-SW-303) and the Frontier Science Projects Program of the Institute of Hydrobiology, Chinese Academy of Sciences (No. 220309).

\section{REFERENCES}

1. Coellingh JP, Rozijn TH, Monfoort CH. Isolation and partial characterization of a basic protein from bovine sperm heads. Biochim Biophys Acta 1969; 188:353-6.

2. Eng LA, Metz CB. Sperm head decondensation by a high molecular weight fraction of sea urchin egg homogenate. J of Exp Zool 1980; 212:159-67.

3. Lohka M, Masui Y. Formation in vitro of sperm pronuclei and mitotic chromosomes induced by amphibian ooplasmic components. Science 1983; 220:719-21.

4. Philpott A, Leno GH, Laskey RA. Sperm decondensation in Xenopus egg cytoplasm is mediated by nucleoplasmin. Cell 1991; 65:569-78.

5. Philpott A, Leno GH. Nucleoplasmin remodels sperm chromatin in Xenopus egg extracts. Cell 1992; 69:759-67.

6. Zhang CM, Clarke PR. Chromatin-dependent nuclear envelope assembly induced by Ran GTPase in Xenopus egg extracts. Science 2000; 288:1429-143.

7. Liu J, Lin HF, Lopez JM, Wolfner MF. Formation of the male pronuclear lamina in Drosophila melanogaster. Developmental Biology 1997; 184:187-96.

8. Burke B, Gerace L. A cell free system to study reassembly of the nuclear envelope at the end of mitosis. Cell 1986; 44:639-52. 
9. Cameron LA, Poccia DL. In vitro development of the sea urchin male pronucleus. Development Biology 1994; 162 : 568-78.

10. Zhao Y, Liu XL, Wu M, Tao W, Zhai ZH. in vitro nuclear reconstitution could be induced in a plant cell-free system. FEBS Letters 2000; 480:208-12.

11. Zhu ZY, Sun YH. Embryonic and genetic manipulation in fish. Cell Research, 2000; 10(1):17-27.

12. Wolenski JS, Hart NH. Scanning electron microscope studies of sperm incorporation into the zebrafish (Brachydanio) egg. J of Exp Zool1987; 243:259-73.

13. Ge W, Jiang YG. Fertilization characteristcs of the sperm of gynogenetic crucian carp (Carassius Auratus Gibelio) in the egg of amphimictic fish. Acta Hydrobiologica Sinica 1985; 9(2):111-5 (in Chinese).

14. Ge W, Jiang YG. Developmental characteristics of sperm nucleus of artificially sex-reversed allogynogenetic crucian carp in amphimictic and gynogenetic ooplasms and their practical significance. Acta Hydrobiologica Sinica 1990; 14(2): 108-13 (in Chinese).

15. Iwamatsu T, Ohta T. Electron microscopic observation on sperm penetration and pronuclear formation in the fish egg. J of Exp Zool 1978; 205:157-80

16. Jiang YG, Liang SC, Chen BD et al. Biological effect of heterologous sperm on gynogenetic offspring in Carassius auratus gibelio. Acta Hydrobiologica Sinica. 1983; 8(1): 1-3 (in Chinese).

17. Yang ZA, Li QH, Wang YF, Gui JF. Comparative investigation on spindle behavior and MPF activity changes during oocyte maturation between genogenetic and amphimictic crucian carp. Cell Research 1999; 9:145-54.

18. Gui JF, Liang SC, Zhu LF, Jiang YG. Discovery of two different reproductive developmental modes of the eggs of artificial multiple tetraploid allogynogenetic gibel carp . Chinese Science Bulletin 1993; 38:332-7. Gui JF.

19. Retrospects and prospects of studies on the mechanism of natural gynogenesis in gibel carp (Carassius auratus gibelio). Science Foundation in China 1997; 5(1):11-6.

20. Gui JF. A unique study system: gynogenetic fish Carassius auratus gibelio. Science Foundation in China 1996; 4 (1):44-6.

21. Zhou L, Wang Y, Gui JF. Genetic evidence for gonochoristic reproduction in gynogenetic gibel carp (Carassius auratus gibelio Bloch) as revealed by RAPD assays. J Mol Evol 2000; 51:498-506.

22. Blow JJ, Laskey RA. Initiation of DNA replication in nuclei and purified DNA by a cell-free extract of Xenopus eggs. Cell 1986; 47:577-87.

23. Blow JJ, Watson JV. Nuclei act as independent and integrated units of replication in a Xenopus cell-free DNA replication system. The EMBO Journal 1987; 6:19972002.

24. Shen SS, Steinhardt RA. Direct measurement of intracellular $\mathrm{pH}$ during metabolic derepression of the sea urchin egg. Nature 1978; 272:253-4.
25. Lopo A, Vacquier VD. The rise and fall of intracellular $\mathrm{pH}$ in sea urchin eggs after fertilization. Nature 1977; 269: 590-2.

26. Zhou L, Wang Y, Gui JF. Molecular analysis of silver crucian carp (Carassius auratus gibelio Bloch) clones by SCAR markers. Aquaculture 2001; 201:219-28.

27. Zhou L, Gui JF. Karyotypic diversity in polyploid gibel carp, Carassius auratus gibelio Bloch. Genetica 2002; 115:223-32.

28. Longo FJ. An ultrastructural study of cross-fertilization (Arbacia? Mytilus ). J Cell Biol 1977; 73:14-26.

29. Ziegler D, Masui Y. Control of chromosome behavior in amphibian oocytes. I.The activity of maturing oocytes inducing chromosome condensation in transplanted brain nuclei. Dev Biol 1973; 35:283-92.

30. Rudak E, Jcobs PA, Yanagimachi R. Direct analysis of the chromosome constitution of human spermatozoa. Nature 1978; 274:911-3.

31. Brown DB, Miskimins WK, Ruddle FH. Partial purification of Xenopus laevis egg extract factor(s) that induce swelling in permeabilized human sperm. J of Exp Zool 1991; 258:263-272.

32. Fan LC, Yang ST, Gui JF. Differential screening and characterization analysis of the egg envelop glycoprotein ZP3 cDNAs between gynogenetic and gonochoristic crucian carp. Cell Research 2001; 11(1):7-27.

33. Wen JJ, Xie J, Liu SG, Gui JF. Differential expression and characteristic analysis of a new gene with WD domains in fish oogenesis. Science in China 2001; 44:541-3.

34. Xie J, Wen JJ, Chen B, Gui JF. Differential gene expression in full-grown oocytes between genogenetic and gonochoristic crucian carps. Gene 2001; 271:109-16.

35. Shi YH, Liu J, Xia JH, Gui JF. Screen for stage-specific expression genes between tail bud stage and heartbeat beginning stage in embryogenesis of gynogenetic gibel carp. Cell Research 2002; 12 (2):133-42.

36. Xie J, Wen JJ, Yang ZA, Wang HY, Gui JF. Cyclin A2 is differentially expressed during oocyte maturation between gynogenetic silver crucian carp and gonochoristic color crucian carp. J of Exp Zool (in press).

37. Krude, T, Jackman M, Pines J, Laskey RA. Cyclin/Cdkdependent initiation of DNA replication in a human cellfree system. Cell 1997; 88:109-19.

38. Sheehan MA, Mills AD, Sleeman AM, Laskey RA, Blow JJ. Steps in the assembly of replication-competent nuclei in a cell-free system from Xenopus eggs. The Journal of Cell Biology 1988; 106:1-12.

39. Okuwaki M, Iwamatsu A, Tsujimoto M, Nagata K. Identification of nucleophosmin/B23, an acidic nucleolar protein, as a stimulatory factor for in vitro replication of adenovirus DNA complexed with viral basic core proteins. J Mol Biol 2001; 311:41-55.

40. Collas P. Formation of the sea urchin male pronucleus in cell-free extracts. Molecular Reproduction and Development. 2000; 56:265-70. 